Version Feb 102007

\title{
Astrometric Orbits for Hipparcos Stochastic Binaries
}

\author{
A. Goldin ${ }^{1}$ \& V.V. Makarov ${ }^{2}$ \\ ${ }^{1}$ Citadel Investment Group, 131 South Dearborn st., Chicago, IL 60603 \\ alexey.goldin@gmail.com \\ ${ }^{2}$ Michelson Science Center, Caltech, 770 S. Wilson Ave., MS 100-22, Pasadena, CA 91125 \\ vvm@caltech.edu
}

\begin{abstract}
Taking advantage of an improved genetic optimization algorithm for fitting unconstrained Kepler orbits to the Hipparcos intermediate astrometric data, we obtain additional orbital solutions for 81 Hipparcos stars with previous stochastic (failed) solutions. The sample includes astrophysically interesting objects, including the old disk wide binary HIP 754, the nearby AGB star HIP 34922 (L2 Pup), and the nearby M2 dwarf HIP 5496 (GJ 54, at 8 pc from the Sun) which has a resolved $\mathrm{M}$ dwarf companion.
\end{abstract}

Subject headings: astrometry — binaries: general

\section{Introduction}

A genetic optimization-based algorithm of unconstrained orbital solutions on Hipparcos Intermediate Astrometry Data (HIAD, ESA 1997) was developed and applied to 1561 stars from the annex of stochastic solutions, resulting in 65 orbiting binaries at the $99 \%$ confidence level (Goldin \& Makarov 2006). Most of the 65 objects had not been known as binaries, although at least two stars on the list of "new" binaries turned out to be parts of a longterm spectroscopic monitoring carried out by other researches (R. Griffin, J. Sperauskas, priv. comm.). Since our first publication about orbiting stochastic stars, we have realized that a simple improvement of the algorithm can be effected if the intermediate astrometric data produced by the two data analysis consortia (NDAC and FAST) are combined prior to running the optimization program, as opposed to our initial decision to run the program 
separately for the NDAC and FAST data points and to compare the final results. Typically, each great circle measurement produced a pair of abscissa data points, one derived by FAST, and the other by NDAC. Our initial disinclination to average the data derived from the same observations was justified by the strong correlation which is underestimated in the catalog. At the same time, some of the astrometric abscissae derived from the same observations differ significantly between the two consortia, betraying unknown model or systematic errors. These errors can be diminished by simple averaging, but the estimated precision can only slightly improve because of the correlated noise. The safest and most conservative approach is to compute the mean values of the common data points, but to adopt the larger of the two formal errors as the expected standard deviation of the result, effectively assuming a correlation of 1 . Thus, our choice of methods and algorithms is driven by the intent to minimize the number of false positives (which can not be precluded completely with these noisy data) and to produce confidence estimates as realistic as possible.

\section{Uncertainties and bias}

Our results are affected by stochastic uncertainties, as well as by considerable bias, especially in the important parameters $a_{0}$ (apparent orbit size) and $e$ (eccentricity). Therefore, it is of crucial importance not only to compute an orbital fit to a set of data points, but also to evaluate the probability distributions for the derived parameters. The one-dimensional nature of Hipparcos measurements, the strong irregularity of observing times and position angles, and the presence of unmodeled disturbances, such as visual companions, all combine to often produce oddly shaped, asymmetric distribution functions. In order to compute realistic precision and bias estimates, we use the parametric bootstrap method (Efron \& Tibshirani 1993).

Our general optimization method is described in (Goldin \& Makarov 2006). First, we

minimize $\chi^{2}$ on abscissae residuals given observed transit times $t_{i}$ and transit times error estimates $\sigma_{i}$. The best fit yields estimated times $t_{e i}$ such that $t_{i}^{j}=t_{e i}^{j}+\sigma_{i} \epsilon_{i}^{j}$, where $\epsilon_{i}^{j}$ are normally distributed normal variables with variance 1 and $j$ is the number of realization. With sufficiently large number of realizations, we build a histogram for each estimated fitting parameter. The expected error of the estimate can be derived from the width of the histogram. In case of zero bias and nearly symmetric distribution, the mode of the distribution, its mean and median are close to value derived from the original data.

As an example, we consider the eccentricity fits produced by our unconstrained optimization algorithm for a subset of 231 stars identified as orbital solutions in the main Hipparcos catalog (ESA 1997). Obviously, for high values of eccentricity the bias on the 
positive side is limited by the hard limit of 0.99 imposed by our program. But if we consider only solutions with small enough eccentricity (less then 0.5 ), we can clearly see that only one solution out of 90 has a negative estimated bias. Only 3 solutions out of these 90 with small eccentricity have a negative estimated bias in semimajor axis $a_{0}$.

Due to the nonlinear mapping of $t_{i}$ to the orbital parameters, the distributions of the mass function and $a_{0}$ may have extremely long tails. Let $V_{\text {est }}$ be the estimated parameter value from the original data, and $V^{j}$ - the estimate from a bootstrap realization $j$. To avoid the unwanted influence of occasional extreme values of $V$ on our error and bias estimate, we define bias via the median, viz., $B(V)=V_{\text {est }}-\operatorname{median}(V)$, and $1 \sigma$-error bars (which can be asymmetric) as $\sigma_{-}(V)=\operatorname{median}(V)-q_{0.16}(V)$ and $\sigma_{+}(V)=q_{0.84}(V)-\operatorname{median}(V)$. where $q_{g}(V)$ is the $g$-quantile of the distribution of $V^{j}$.

For sufficiently small errors and sufficiently large number of data points, it is possible to estimate the unbiased value of $V_{\text {unbiased }}=V_{\text {est }}-B(V)$. We do not attempt this correction for the stochastic solutions considered in this work. For the 231 stars with catalogued Hipparcos orbital solutions, one can see from Fig. 1 that the bias of $e$ is consistently positive if $e_{e s t}$ is not too large. Note that our eccentricity estimation is restricted to the interval [0, 0.99], but the bias estimation is restricted only at the upper limit. It is likely that this bias is responsible for the abundance of solution with eccentricities close to the cutoff value of 0.99.

\section{Orbits}

An automated run of the modified optimization algorithm on all 1561 stochastic stars in the Hipparcos catalog resulted in about 200 new solutions with estimated confidence levels greater than $99 \%$. The meaning of this confidence estimation is that the HIAD data for these stars is perturbed in such a way that the apparent positions are consistent with the specified orbital model, with the probability of a random occurrence of such data for a single, unperturbed star less than $1 \%$. This is not to say that the given solutions are accurate to $99 \%$, or even that the stars are $99 \%$-probability binaries. Indeed, we find that roughly one half of all automated solutions from the stochastic set have eccentricities equal to 0.99 , and large orbital sizes. This unexpected set of failed fits indicates that undetected

orbital motion in our somewhat idealized model only accounts for a fraction of stochastic solutions. Fabricius \& Makarov (2000) demonstrated that a number of stochastic stars are visual, resolved binaries with negligible orbital motion, where the secondary companion was not listed in the Hipparcos input catalog, or given at an incorrect position. Using correct initial assumptions about the relative position of components in binary and multiple 


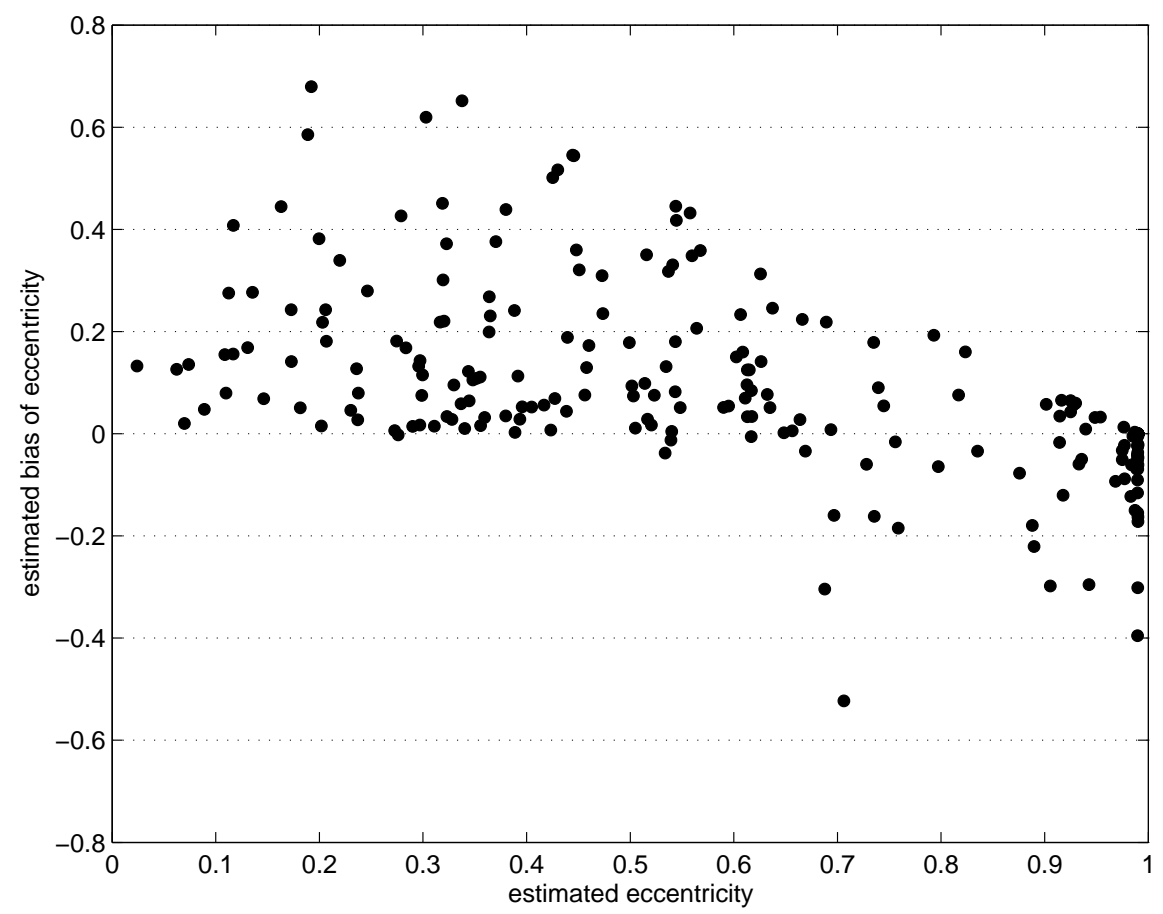

Fig. 1.- Orbital eccentricity and bias of eccentricity estimated by the parametric bootstrap method for 231 binary stars with orbital solutions in the Hipparcos catalog. 
systems can yield regular, high-quality astrometric solutions by a straightforward leastsquares adjustment. But the model of fixed visual companions is another simplification, bound to meet limited success with the real objects presenting a host of complicating features. For example, a visual companion can have a detectable motion around the primary, resulting in an effectively different proper motion; the inner pair in a hierarchical triple system can be orbiting; one or both components of an orbiting pair can be variable stars evoking the variability-imposed motion (VIM) effect on the observed photocenter (Pourbaix 2001). For red stars, the data itself may need recalibration, since the assumed color was in many cases biased (Platais et al. 2000). In principle, increasingly sophisticated algorithms can be created to deal with such cases; in practice though, the optimization problem becomes cumbersome with the available data because we are quickly running out of data points with each additional nonlinear fitting parameter.

At this point, we simply rejected all the spurious-looking solutions with extreme eccentricities. The remaining 81 fits are specified in Table11. Orbital fits for the stars published in our preceding paper (Goldin \& Makarov 2006) are also omitted, even if they differ for some stars. The estimated astrometric parameters, period $P$ in days, eccentricity $e$, periastron time $T_{0}$ in days, longitude of the ascending node $\omega$ in degrees, position angle of the node $\Omega$ in degrees, and inclination $i$ (zero for face-on orbits) in degrees are specified in the table. These data are based on 1000 genetic optimization trials for each object. Confidence limits for each fitting parameter are computed at several confidence levels from the set of 1000 simulations, but only the 84 th and 16 th percentiles are used to compute the robust uncertainty limits in Table 1, which correspond to the $\pm 1 \sigma$ interval of $N(m, \sigma)$. These intervals provide a minimalistic estimation of the parameter uncertainties, but carry no information about the shape of the distribution, which can be strongly non-Gaussian, as discussed in the previous paragraph. Our solutions are perfectly unconstrained, and are not based on any prior information except the HIAD.

A literature search for the stars listed in Table 1 reveals that three objects have been known as spectroscopic binaries from precision radial velocity observations. Two of them have accurate spectroscopic orbits published, viz., HIP $34164=$ G 108-53 Latham et al. 2002) and HIP $\mathbf{6 7 4 8 0}=\mathbf{e}$ Boo (Griffin 1985). Both stars were also re-analyzed by Jancart et al. (2005), whose computations were also based on the HIAD, but the essential nonlinear parameters $(P, e$ and $\omega)$ were fixed at their spectroscopic values. The cited spectroscopic data, our unconstrained fits and constrained astrometric solutions from (Jancart et al. 2005) are specified in Table 2 for comparison. Encouragingly, our periods come out fairly close (within 1-2 $\sigma$ ) to the more accurate spectroscopic estimates, and the eccentricities are even closer to the mark for both stars. The unconstrained estimates of $\omega$ probably include the uncertainty of $180^{\circ}$ in both cases. Therefore, we do not see any reasons to be concerned with 
our method and results from the comparison with the spectroscopic data. The constrained solutions for the apparent orbit size $a_{0}$ and inclination $i$ from (Jancart et al. 2005) are much stronger than ours, because fixing the troublesome nonlinear parameters (especially, e) simplifies the multidimensional $\chi^{2}$ surface and removes many of the valley-like local minima. For HIP 34164, our estimate of $i$ is comfortably close to the constrained parameter. The corrected mass function, $M_{2}^{3} /\left(M_{1}+M_{2}\right)^{2}$, is fairly high at $0.095 M_{\odot}$ for this high proper motion, moderately metal-poor $([\mathrm{Fe} / \mathrm{H}]=-0.68)$ system.

For the star HIP 67480 (e Boo), our unconstrained fit and the constrained solution from (Jancart et al. 2005) produce similar orbit sizes, but significantly different inclination angles. The former value of $i$ is brought into agreement with the small radial velocity amplitude reported by Griffin (1985) for this K4III star and the spectroscopic $a_{1} \sin i \approx 14.1 \mathrm{Gm}$. Our unconstrained solution would imply a companion of moderate mass in the absence of a spectroscopic data, whereas we are probably dealing with an orbit seen almost face-on.

Finally, the F7V star HIP $\mathbf{1 1 0 7 8 5}$ = HD 212754 has been listed by Griffin \& Suchkov (2003) as a single-lined spectroscopic binary, but without orbital solution. This star has been drawing considerable attention of observers since it was identified as a likely member of the Hyades moving group. Our solution yields an almost edge-on orbit of size $a_{0} \approx 0.43$ AU. From this, and the period $P=878 \mathrm{~d}$, we conclude that the mass ration $M_{2} / M_{1}$ may be fairly small $(\simeq 1 / 5)$.

The Geneva-Copenhagen (GC) spectroscopic survey of Hipparcos solar-type stars (Nordström et al. 2004) provides an independent verification of our results. Most of the stars in this survey obtained only a few radial velocity (RV) measurements, which was insufficient to derive an orbital fit, but sufficient to establish the fact of spectroscopic binarity. For stars with more than one measurement the probability is specified that the RV has not changed significantly relative to the estimated measurement error. These probability estimates are useful to make sure that most of our orbital solutions indeed correspond to binary systems, although the quality of our solutions can not be verified. We find 15 stars in common between Table 1 and GC with estimated probabilities of constant RV, viz., HIP 754, 1768, 3645, 12726, 17482, 32307, 49638, 56447, 64790, 84696, 101430, 110785, 113177, and 113699. All these probabilities are zero or very small in GC. We conclude that the incidence of spectroscopic binarity among the new solutions is probably high.

The stars HIP 3645, 43067, 49638, 50567, 84696, 109095 are listed in (Makarov \& Kaplan 2005) as astrometric binaries whose long-term proper motions from the Tycho-2 catalog are statistically different from the short-term Hipparcos proper motion. Such discrepancies are usually caused by significant long-period orbital motion. The fitted periods for these stars are indeed at the upper end of the range in Table 1, exceeding 3-4 years. Admittedly, this 
is not a clean check on the quality and reliability of our solutions, because the stochastic proper motions in Hipparcos can be perturbed more than their formal errors manifest.

The data in Table 1 indicate that our method is especially sensitive for orbital periods between 1 and 4 years, with relatively few shorter or longer periods detected. The smallest orbital size appears to be 5 mas. It is difficult to derive robust orbital solutions for smaller apparent orbits even for the brightest stars, where the typical single measurement precision is comparable to or less than 5 mas. This is an interesting fact pertaining to the scarcity of new low-mass companions detected from the HIAD, and the complete lack of planet signatures. Rather than a hint of lower than expected precision of the data, we consider it to be a still poorly understood feature of the given nonlinear optimization problem, which has been empirically found to have a worse condition than what could be naively expected from the number of data points and the number of fitting parameters (see also Pourbaix 2001).

The orbital fits for a few stars in Table 1 are so definite and robust, that one may wonder why they were not resolved by the Hipparcos data analysis team and placed in the annex of orbital solutions. Fig. 2 shows the reconstructed orbit and the averaged observations for one of these stars, HIP 113699. Our fit yields an orbital period of nearly 2 years and an apparent motion of $a_{0}=9.5$ mas for this poorly investigated G0 dwarf. The star was part of the systematic attempt to resolve Hipparcos stochastic binaries with speckle interferometry by Mason et al. (2001), which was unsuccessful. The estimated orbit size in combination with the parallax (23 mas) indicates that the companion may be much fainter than the primary. The estimated inclination suggests that the star should be easily detectable as SB1 in RV measurements.

\section{Objects of note}

HIP $5496=$ GJ 54 is a nearby M dwarf at a distance of $\approx 8$ pc from the Sun. The stochastic Hipparcos parallax $123 \pm 8$ mas is somewhat uncertain, but a recent groundbased observation puts the star confidently within 10 pc (Henry et al. 2006). Golimowski

et al. (2004) resolved a visual companion to this star using HST NICMOS observations and found a magnitude difference of $\approx 1$ mag between the components. Our solution for this star is relatively robust, with a period of $427(+9-8)$ d and apparent semimajor axis $a_{0}=19.0(+7.4,-2.1)$ mas. The small orbital size for this nearby star seems to suggest a lowmass companion, perhaps a brown dwarf, raising doubts that the orbiting companion and the visual are the same one. However, the provisional data from (Henry et al. 2006) confirm our orbital solution, estimating the period at $\approx 1.1$ year and the apparent orbit of the photocenter at 16 mas. Obviously, the companion is bright enough (magnitude difference of 1.04 in $V$ ) to 


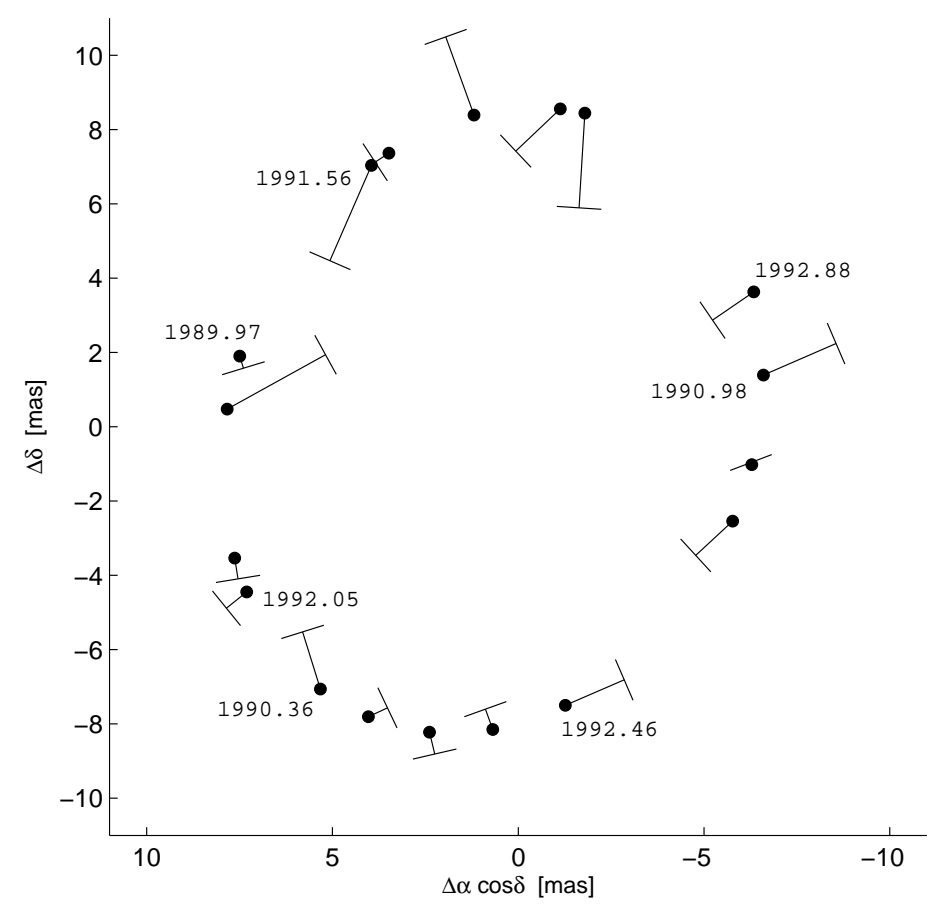

Fig. 2. - The apparent orbit of the photocenter of the new binary HIP 113699. The observed abscissa residuals are shown with straight segments connecting the estimated orbital positions (indicated with black circles) and the actual observations. The mean epoch positions of the Hipparcos grid of slits is marked with short straight segments. The $\chi^{2}$-adjusted parameters (slightly different from those specified in Table 1) are: $P=667 \mathrm{~d}, e=0.1$, $T_{0}=216 \mathrm{~d}, i=36.4^{\circ}, a_{0}=8.7$ mas, $\omega=97^{\circ}$. 
pull the photocenter closer to the center of mass, making the observed ellipse much smaller than the true one. A simple calculation shows that for a plausible total mass of $0.5 M_{\odot}$, the full orbit is $a=0.88 \mathrm{AU}$, and since the observed photocenter orbit is $0.15 \mathrm{AU}$, the primary's orbit is $0.40 \mathrm{AU}$, and the mass ratio $q=M_{2} / M_{1}=0.83$. Thus, the Hipparcos measurements and our orbital solution are consistent with the known properties of the resolved companion. What remains puzzling is the overall brightness of the system. According to (Henry et al. 2006), the magnitudes are $V=9.82, I=7.32, J=6.00$, and $K=5.13$ mag. This compares well with the accurate photometry from (Koen et al. 2002), $V=9.812 \pm 0.008$ and $I=7.357 \pm 0.009$ mag. Fig. 3 depicts the well-defined empirical main sequence of nearby dwarfs in the $M_{K}$ vs. $V-K$ axes from (Henry et al. 2004) (lower curve). The short curled curves with beads on them indicate the joint photometry of unresolved main-sequence binaries on a grid of primary absolute magnitudes, for magnitude differences between the companions of $\Delta V=+\infty, 6,3,2,1$, and 0 mag. The latter value corresponds to the case of two equally bright companions (twins). With the trigonometric parallax determined by Hipparcos, the system is much brighter in $K$ than the upper envelope of possible magnitudes for binary stars. The parallax measured by Henry et al. (2006) (141.2 \pm 3.4 mas) brings it closer to the range of normal magnitudes, but interestingly, our updated astrometric solution for parallax is close to the original Hipparcos value. Henry et al. (2006) contend that this system represents almost the worst case, when the orbital period is close to one year, and it becomes difficult to distinguish between the parallactic and orbital ellipses. The covariance analysis technique on linearized condition equations described in (Goldin \& Makarov 2006) is useful to estimate the degree of possible perturbation of the parallax solution. We compute a significant correlation coefficient of -0.24 for the pair of parameters $a_{0}$ and $\Pi$. It can be suspected that the trigonometric parallax is underestimated; therefore, the orbit size may be slightly overestimated. We also find, surprisingly, large positive correlations between $\Pi$ and the angles $\omega$ and $\Omega$ of 0.57 and 0.61 , respectively. It follows, that the actual difficulty with this star is that the parallax determination is strongly coupled with the orientation of the orbit, which is poorly constrained by our data.

HIP $34922=\mathbf{L}_{2}$ Pup is probably the nearest asymptotic giant branch (AGB) star to the Sun. AGB stars are in the terminal stages of stellar evolution characterized by massive loss of mass. By virtue of its large-scale, stable variability of brightness, the star has been classified as semiregular pulsating variable. Our unconstrained fit for this star produced a robust solution with a period of $141(+2,-1)$ days, which is identical to the variability period of $140.6 \mathrm{~d}$. This prompted us to investigate if the star could be a Variability-Imposed-Motion (VIM) binary, whose photocenters move along the line connecting the components in phase with the variability, because of the shift of the center of light. A general VIM fit produced a meagre reduction in the residual $\chi^{2}$, ruling out a simple VIM model. Another possibility of 


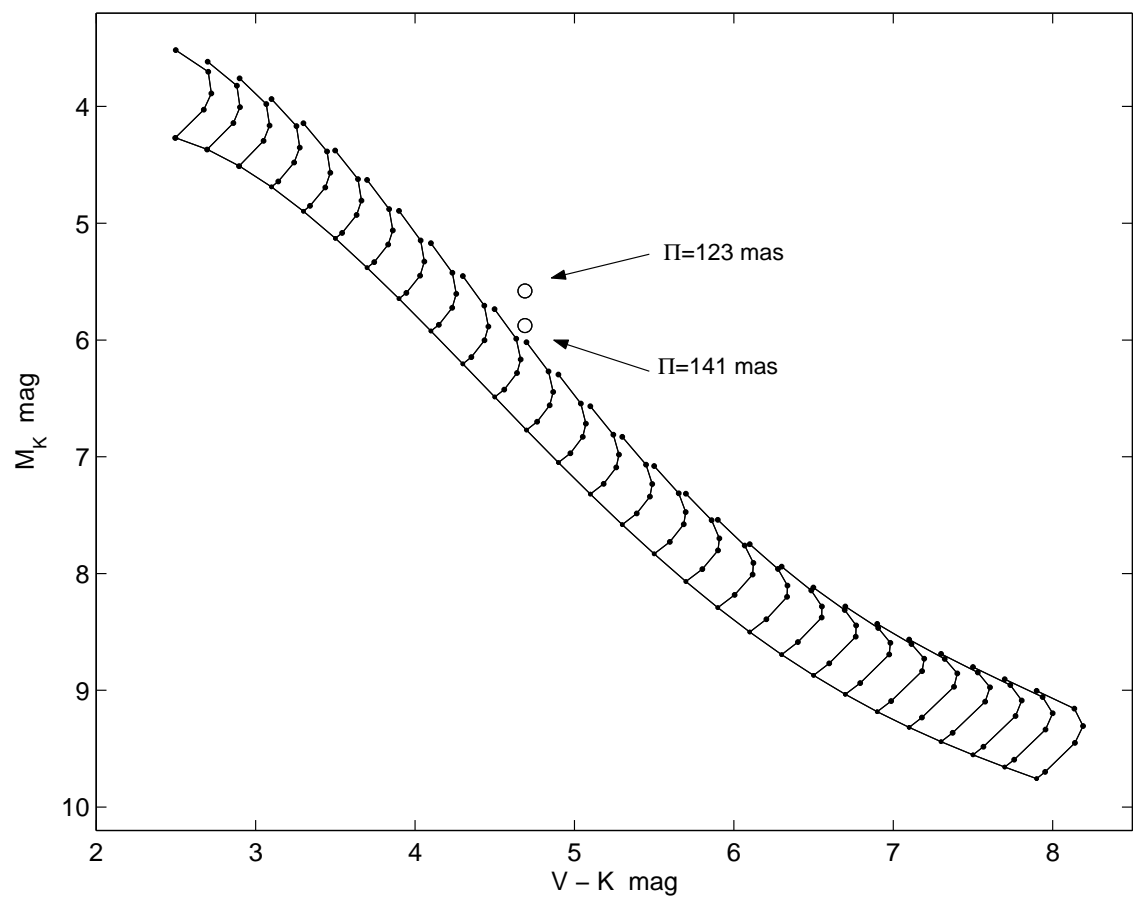

Fig. 3.- The empirical main sequence of field dwarfs from (Henry et al. 2004) (lower curve) and the location of binaries with joint photometric magnitudes shown with short curves and dots for $\Delta V=0$ (upper end points), 1, 2, 3, 6 and $+\infty$ mag. The loci of the star GJ 54 are indicated with open circles for two parallaxes, 123 and 141 mas. 
a spurious astrometric fit is predicated by the incorrect $V-I$ color indeces that had been assumed for some of the red stars in the data reduction. This could generate significant errors in color-dependent calibration terms, as explained in detail in (Platais et al. 2000). For this star, however, the assumed color $(V-I=4.18)$ was not too different from the color derived from subsequent photometric observations $(V-I=3.51)$, and the calibration error is not expected to exceed 1 mas, which is much smaller than the fitted orbit $(9.5 \pm$ $[+4.1-1.3])$. Thus, $\mathrm{L}_{2}$ Pup is quite likely a real orbiting binary. Jura et al. (2002) obtained and studied mid-infrared images of $\mathrm{L}_{2}$ Pup and found an extended asymmetric morphology of the shell. The asymmetry was later confirmed from the interferometric observations by Ireland et al. (2004). Lebzelter et al. (2005) detected periodic variation of radial velocity, but unfortunately, the small number of data points does not permit to estimate the phase shift between the light curve and the RV curve. We propose that the stable period of the variability curve, the recent dimming of the object and other peculiar properties of the object can be explained by a bright companion orbiting the primary AGB star and getting periodically obscured by its thick dust shell. A more detailed discussion of this system will be published elsewhere.

HIP $754=$ HD 471 has been netted in earlier searches for field subdwarfs because of its considerable proper motion (e.g., Sandage \& Kowal 1986). However, the star is moderately metal poor at $[\mathrm{Fe} / \mathrm{H}]=-0.16$ (Nordström et al. 2004), has a small Galactic eccentricity of 0.11, and its maximum excursion from the Galactic plane is limited to 258 pc (Allen et al. 2000). Thus, it is not a subdwarf, but Allen et al. argue that it is one of the oldest known wide binaries with a photometrically estimated age of $11.6 \mathrm{Gyr}$. The wide companion is separated by a little less than $30^{\prime \prime}$. This is definitely a physical pair, with proper motions from the extensive Nomad catalog (Zacharias et al. 2003) $\left(\mu_{\alpha *}, \mu_{\delta}\right)=(174.9,-146.7) \pm(1.8,1.3)$

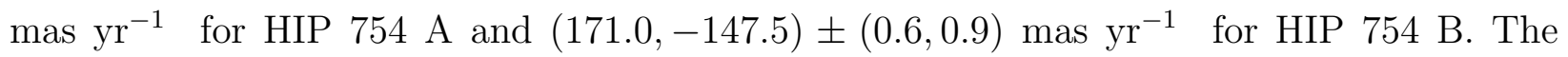
wide companion has a separation of at least $1200 \mathrm{AU}$, and a period $\gtrsim 34000 \mathrm{yr}$. Dynamical evolution and the origin of old wide binaries are interesting issues motivating the long-term effort to increase the sample of such systems and to collect more observations. Given the considerable attention to this star in the literature, it is surprising that binarity of the primary in this pair has remained unnoticed. Our solution suggests a fairly large apparent orbit of $9.8 \pm(+3.9,-1.5)$ mas and a nearly edge-on position, explaining the large scatter of radial velocities observed in (Nordström et al. 2004). The corrected parallax is $24.8 \pm 1.0$ mas. Assuming a total mass of $1.5 M_{\odot}$, the mass of the primary is roughly $1.1 M_{\odot}$ and the secondary $0.4 M_{\odot}$. Tokovinin et al. (2006) argue that new stars do not form in short-period binaries, and we mostly owe the presence of spectroscopic binaries with periods less than 10 $\mathrm{d}$ to the dynamical interaction between the inner pair and the remote tertiary companion in hierarchical multiple systems, which takes giga-years to shrink the inner orbit, and perhaps, 
to eventually merge the close pair. The HIP 754 system, if it really is very old, appears to be an example to the contrary, when the tertiary failed for some reasons to shrink the close inner binary.

\section{Facility: HIPPARCOS}

The research described in this paper was in part carried out at the Jet Propulsion Laboratory, California Institute of Technology, under a contract with the National Aeronautics and Space Administration. This research has made use of the SIMBAD database, operated at CDS, Strasbourg, France. 


\section{REFERENCES}

Allen, C., Poveda, A., Herrera, M.A. 2000, A\&A, 356, 529

Efron B, Tibshirani, R. 1993, An Introduction to the Bootstap. Chapman \& Hall/CRC.

ESA, 1997, The Hipparcos and Tycho Catalogues, ESA SP-1200

Fabricius, C., \& Makarov, V.V., 2000, A\&AS, 144, 45

Goldin, A., Makarov, V.V. 2006, ApJS, 166, 341

Golimowski, D.A., et al., 2004, AJ, 128, 1733

Griffin, R.F. 1985, JA\&A, 6, 77

Griffin, R.F., Suchkov, A.A. 2003, ApJS, 147, 103

Ireland, M., et al. 2004, MNRAS, 350, 365

Jancart, S., et al. 2005, A\&A, 442, 365

Jura, M., Chen, C, Plavchan, P. 2002, ApJ, 569, 964

Henry, T.J., et al. 2004, AJ, 128, 2460

Henry, T.J., et al. 2006, AJ, 132, 2360

Koen, C., et al. 2002, MNRAS, 334, 20

Latham, D.W., et al., 2002, AJ, 124, 1144

Lebzelter, T., et al. 2005, A\&A, 431, 623

Makarov, V.V., \& Kaplan, G,H., 2005, AJ, 129, 2420

Mason, B.D., et al. 2001, AJ, 121, 3224

Nordström et al., 2004, A\&A, 418, 989

Platais, I., et al. 2000, A\&A, 397, 997

Pourbaix, D. 2001, A\&A, 369, 22L

Sandage, A., Kowal, C. 1986, AJ, 91, 1140

Tokovinin, A., et al., 2006, A\&A, 450, 681 
Zacharias, N., Monet, D. G., Levine, S. E., Urban, S. E., Gaume, R., Wycoff, G. L. 2004, BAAS, 205, 4815 


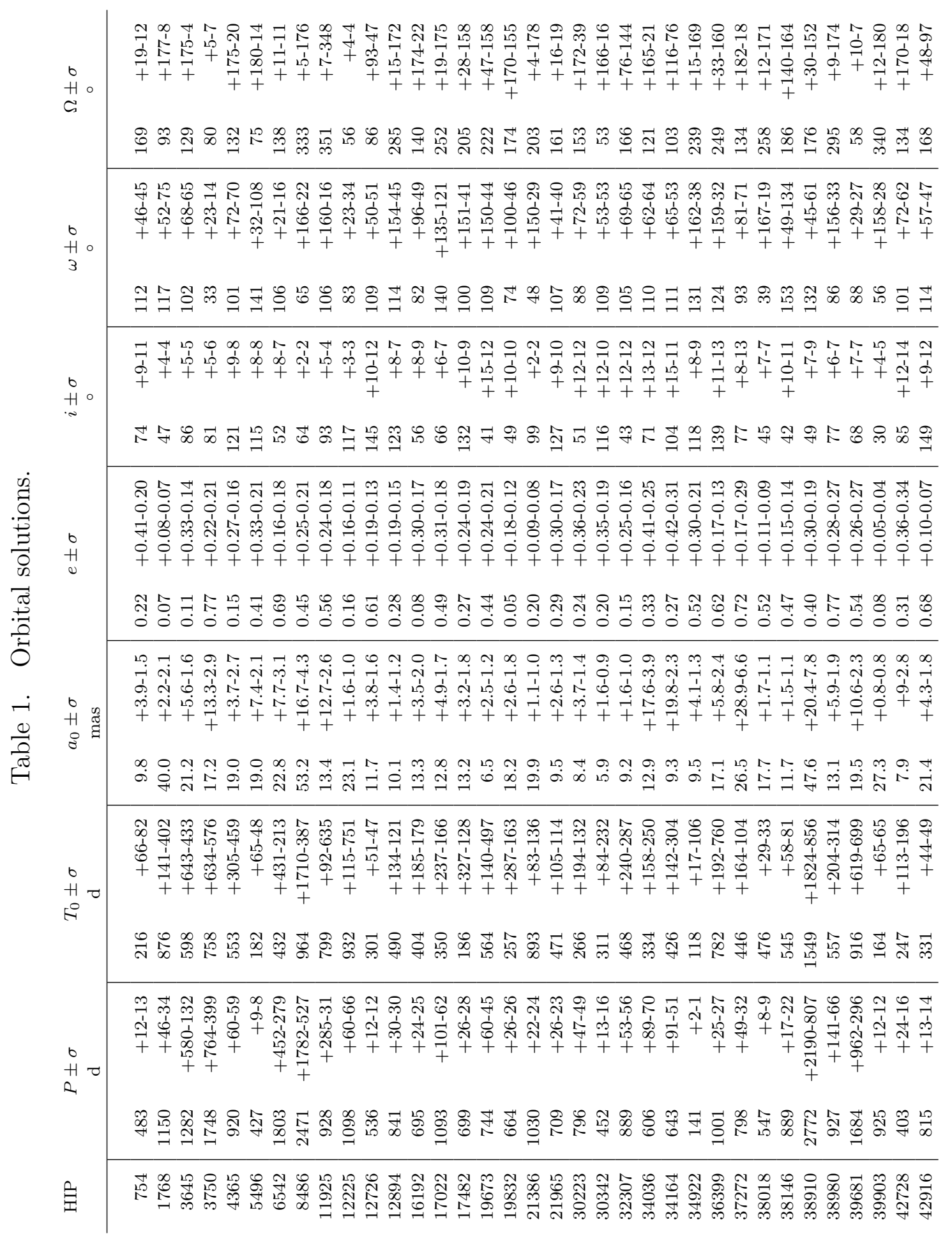




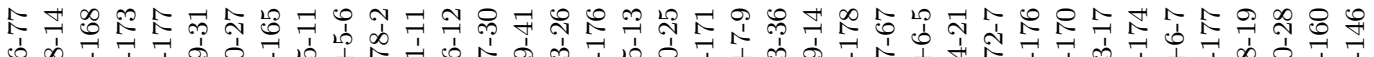

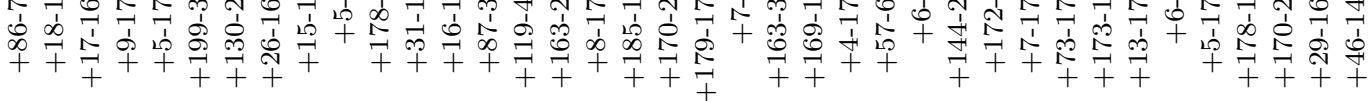

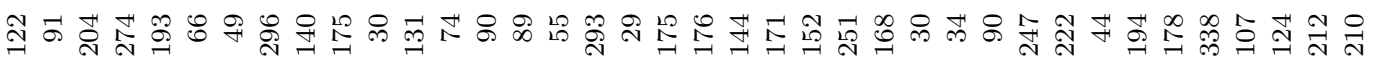

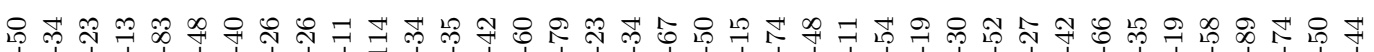

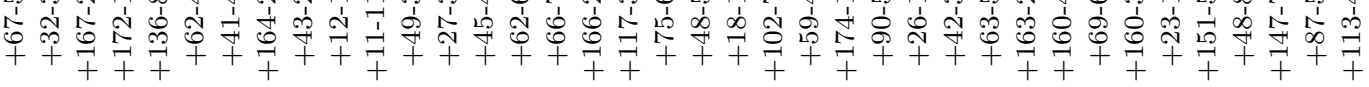

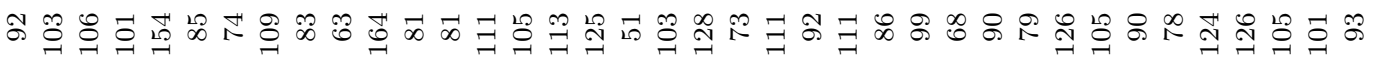

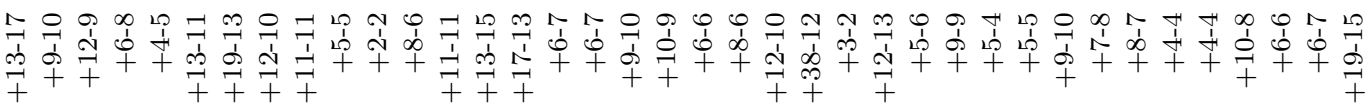

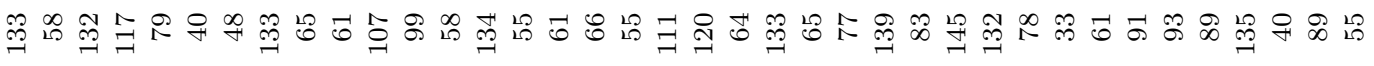

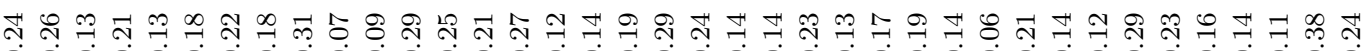

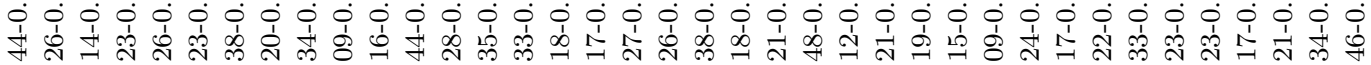

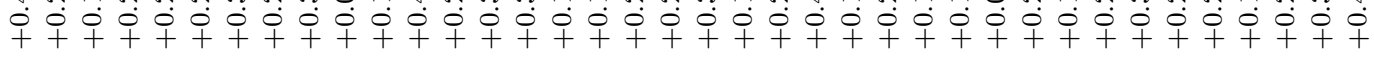

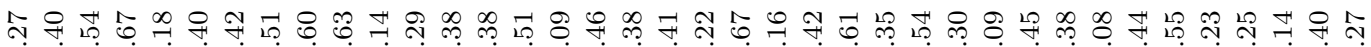

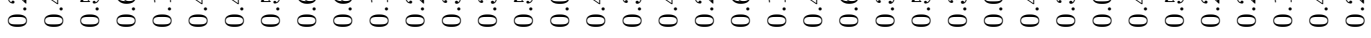

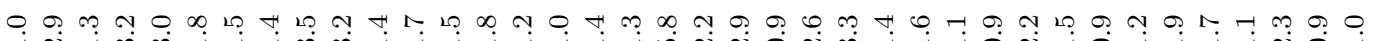

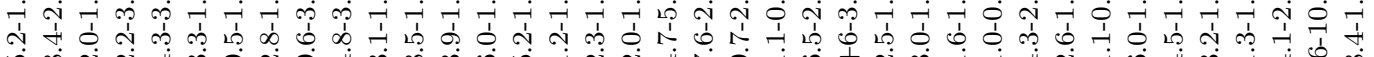

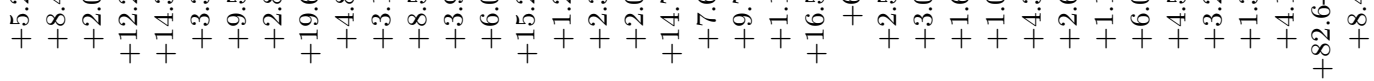

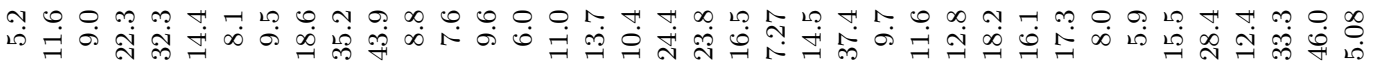

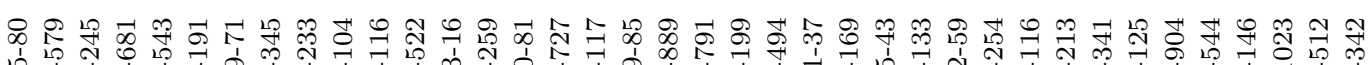

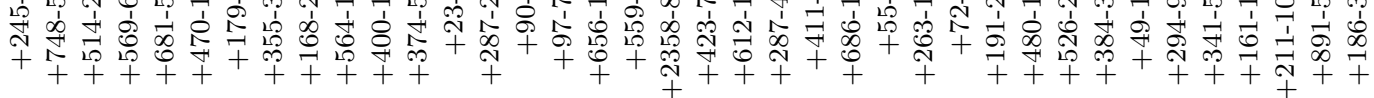

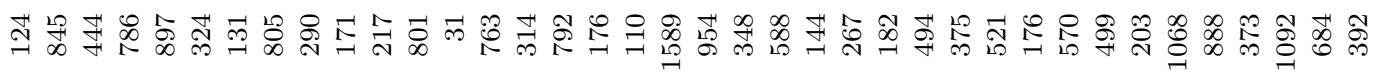

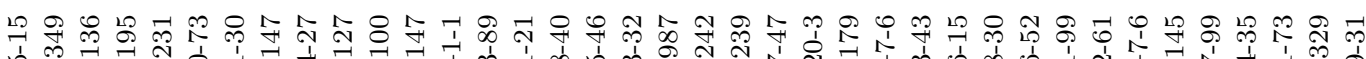

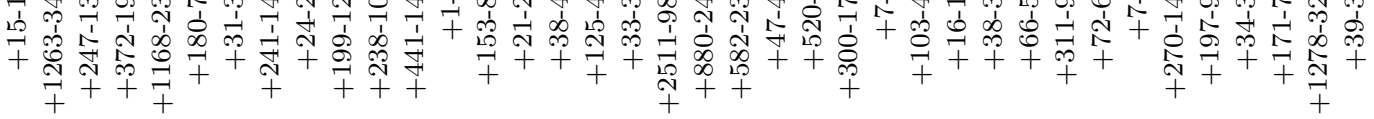

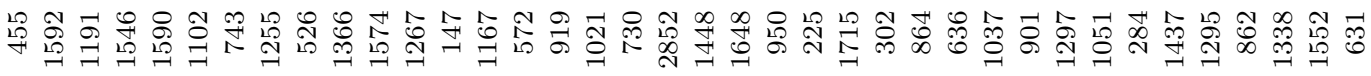

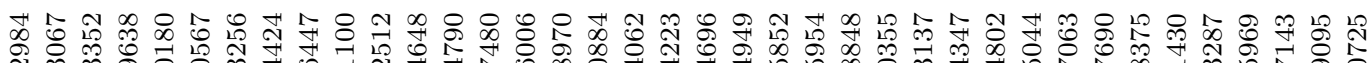

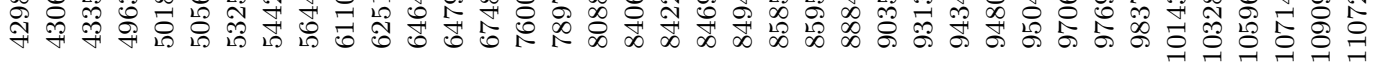




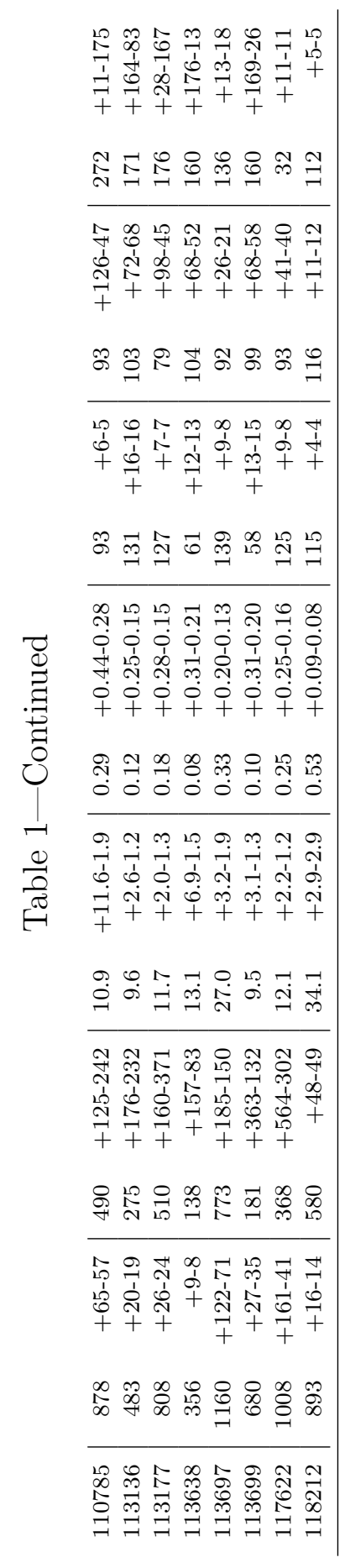


Table 2. Comparison of astrometric fits with known spectroscopic orbits and constrained solutions.

\begin{tabular}{|c|c|c|}
\hline Element & HIP 34164 & HIP 67480 \\
\hline \multicolumn{3}{|l|}{ Spectroscopic } \\
\hline$P[\mathrm{~d}]$ & $612.3 \pm 3.0$ & $944 \pm 8$ \\
\hline$e$ & $0.273 \pm 0.015$ & $0.41 \pm 0.09$ \\
\hline$f M$ & $0.0826 \pm 0.0061$ & $0.00013 \pm 0.00005$ \\
\hline$\omega\left[^{\circ}\right]$ & $248.9 \pm 3.7$ & $359 \pm 15$ \\
\hline$a_{1} \sin i[\mathrm{Gm}]$ & $91.9 \pm 2.4$ & $14.1 \pm 1.9$ \\
\hline \multicolumn{3}{|c|}{ Astrometric, unconstrained (this work) } \\
\hline$P[\mathrm{~d}]$ & $643_{-51}^{+91}$ & $1167_{-89}^{+153}$ \\
\hline$e$ & $0.27_{-0.31}^{+0.41}$ & $0.38_{-0.21}^{+0.35}$ \\
\hline$i\left[^{\circ}\right]$ & $104_{-11}^{+15}$ & $134_{-15}^{+13}$ \\
\hline$\omega\left[^{\circ}\right]$ & $111_{-53}^{+65}$ & $111_{-42}^{+45}$ \\
\hline$a_{0}[\mathrm{mas}]$ & $9.3_{-2.3}^{+19.8}$ & $9.6_{-1.8}^{+6.0}$ \\
\hline \multicolumn{3}{|c|}{ Astrometric, constrained (Jancart et al. 2005). } \\
\hline$i\left[^{\circ}\right]$ & $107.4 \pm 8.5$ & $174.0 \pm 0.5$ \\
\hline$a_{0}$ [mas] & $8.77 \pm 0.96$ & $7.3 \pm 0.9$ \\
\hline
\end{tabular}

Note. - Spectroscopic data from Latham et al. 2002 and Griffin 1985) 